\title{
Phosphorylation of Rab37 by protein kinase C alpha inhibits the exocytosis function and metastasis suppression activity of Rab37
}

\author{
Hong-Tai Tzeng ${ }^{1}$, Tsung-Hsin Li ${ }^{1}$, Yen-An Tang ${ }^{1,2}$, Chung-Han Tsai ${ }^{3}$, Pei-Jung Frank \\ Lu $^{4}$, Wu-Wei Lai ${ }^{5}$, Chi-Wu Chiang ${ }^{3,6}$ and Yi-Ching Wang ${ }^{1,3}$ \\ ${ }^{1}$ Department of Pharmacology, College of Medicine, National Cheng Kung University, Tainan, Taiwan \\ ${ }^{2}$ Cancer Therapeutics and Stratified Oncology, Genome Institute of Singapore, Agency for Science, Technology and Research \\ (A*STAR), Singapore \\ ${ }^{3}$ Institute of Basic Medical Sciences, College of Medicine, National Cheng Kung University, Tainan, Taiwan \\ ${ }^{4}$ Institute of Clinical Medicine, College of Medicine, National Cheng Kung University, Tainan, Taiwan \\ ${ }^{5}$ Division of Thoracic Surgery, Department of Surgery, National Cheng Kung University Hospital, College of Medicine, National \\ Cheng Kung University, Tainan, Taiwan \\ ${ }^{6}$ Institute of Molecular Medicine, College of Medicine, National Cheng Kung University, Tainan, Taiwan \\ Correspondence to: Yi-Ching Wang, email: ycw5798@mail.ncku.edu.tw \\ Chi-Wu Chiang, email: chiangcw@mail.ncku.edu.tw
}

Keywords: Rab37, PKCa, exocytosis, metastasis, prognosis

Received: May 19, 2017 Accepted: September 05, 2017 Published: September 18, 2017

Copyright: Tzeng et al. This is an open-access article distributed under the terms of the Creative Commons Attribution License 3.0 (CC BY 3.0), which permits unrestricted use, distribution, and reproduction in any medium, provided the original author and source are credited.

\section{ABSTRACT}

We previously identified a novel Rab small GTPase protein, Rab37, which plays a critical role in regulating exocytosis of secreted glycoproteins, tissue inhibitor of metalloproteinases 1 (TIMP1) to suppress lung cancer metastasis. Patients with preserved Rab37 protein expression were associated with better prognosis. However, a significant number of the patients with preserved Rab37 expression showed poor survival. In addition, the molecular mechanism for the regulation of Rab37mediated exocytosis remained to be further identified. Therefore, we investigated the molecular mechanism underlying the dysregulation of Rab37-mediated exocytosis and metastasis suppression. Here, we report a novel mechanism for Rab37 inactivation by phosphorylation. Lung cancer patients with preserved Rab37, low TIMP1, and high PKCa expression profile correlate with worse progression-free survival examined by Kaplan-Meier survival, suggesting that PKCa overexpression leads to dysfunction of Rab37. This PKCa-Rab37-TIMP1 expression profile predicts the poor outcome by multivariate Cox regression analysis. We also show that Rab37 is phosphorylated by protein kinase $\mathrm{Ca}$ ( $\mathrm{PKCa}$ ) at threonine 172 (T172), leading to attenuation of its GTPbound state, and impairment of the Rab37-mediated exocytosis of TIMP1, and thus reduces its suppression activity on lung cancer cell motility. We further demonstrate that PKCa reduces vesicle colocalization of Rab37 and TIMP1, and therefore inhibits Rab37-mediated TIMP1 trafficking. Moreover, Phospho-mimetic aspartate substitution mutant T172D of Rab37 significantly promotes tumor metastasis in vivo. Our findings reveal a novel regulation of Rab37 activity by PKCa-mediated phosphorylation which inhibits exocytic transport of TIMP1 and thereby enhances lung tumor metastasis.

\section{INTRODUCTION}

Rab small GTPases consist of the largest subgroup of Ras superfamily and function as regulators of intracellular vesicle transport, such as endocytosis, receptor recycling and exocytosis [1-3]. Crosstalk between complex networks of Rab and effector proteins, including kinases, phosphatases, sorting or tethering adaptors, and motors ensures precise regulation of vesicle traffic [4-6]. All Rab GTPases have consensus motifs to bind GDP (inactive 
form) and switch to active form when bound to GTP [7]. Notably, emerging evidence show that dysregulated Rabmediated transport pathways and aberrant expression of Rab GTPases are implicated in tumorigenesis. Indeed, overexpression of Rab2A, Rab3D, Rab8, Rab11, Rab21, Rab23, Rab25, Rab27B and Rab35 promotes tumor cell migration and invasion [8-16].

We have previously cloned human Rab37 and found that Rab37 mRNA was down-regulated by promoter hypermethylation in non-small cell lung cancer patients. The low level of Rab37 mRNA expression was associated with tumor metastasis [17]. Therefore, we hypothesize that Rab37 acts as a tumor suppressor in lung cancer. We have identified TIMP1 and TSP1 as the potential trafficking cargos of Rab37 by secretome analysis [18]. We further demonstrate that expression of Rab37 suppresses tumor progression by mediating exocytosis of TIMP1 and TSP1 to inactivate extracellular metalloproteinase 9 and to inhibit tumor neovasculature respectively $[18,19]$. Since the sophisticated regulation of Rab activity is important for spatiotemporal control of vesicle traffic, it remains elusive whether phosphorylation modification of Rab37 regulates its functions in exocytosis and tumorigenesis. In this report, we show that Rab37 phosphorylation by PKC $\alpha$ inactivates Rab37-mediated exocytic transport by decreasing GTP-bound active form and thereby enhances lung tumor invasion and metastasis.

\section{RESULTS}

\section{Clinical impacts of inverse correlation of PKCa/ TIMP1 expression}

We previously reported that preserved expressions of metastatic suppressor Rab37 and its cargo, TIMP1, were associated with better prognosis [18]. However, a subgroup of lung cancer patients with preserved Rab37 level was linked to high mortality. Therefore, we hypothesized that the patients with poor prognosis but preserved Rab37 expression may result from the dysfunction of Rab37 in their tumor tissues. Since members of PKC family regulate physiological function of some Rab GTPases by phosphorylation [20, 21], a panel of PKC inhibitors was used to screen for kinase of Rab37 (Supplementary Figure 1A). Additional gene manipulation experiments showed that PKC $\alpha$ was the candidate kinase for Rab37 phosphorylation (see below). We therefore tested whether Rab37 dysregulation was associated with PKC $\alpha$ level in clinical lung cancer specimens. We examined Rab37, TIMP1 and PKC $\alpha$ expression patterns in tumor specimens from 158 lung cancer patients and assigned protein expression levels into preserved, overexpressed and low expression by immunohistochemical analysis (Figure 1A). A total of $107(67.7 \%)$ patients showed preserved Rab37 protein expression (Figure 1B and Table 1). We then performed stratification analyses for TIMP1 and
PKC $\alpha$ expression in this subgroup of 107 patients with preserved Rab37 expression. Strikingly, we observed a strong inverse correlation between TIMP1 and PKC $\alpha$ (73\%, $p<0.0001$; Figure 1B and Table 1). Patients with preserved Rab37 expression and low TIMP1 expression were significantly associated with PKC $\alpha$ overexpression, supporting our hypothesis that PKC $\alpha$ overexpression leads to dysfunction of Rab37.

To determine the prognostic effects of Rab37, TIMP1 and PKC $\alpha$ expression, survival curves were generated using the Kaplan-Meier method. Patients with preserved Rab37 and TIMP1 expression, and low $\mathrm{PKC} \alpha$ expression had better overall survival and diseasefree survival, while patients with preserved Rab37, low TIMP1, and high PKC $\alpha$ displayed significantly worse outcome $(P=0.001$ for overall survival; $P=$ 0.001 for disease-free survival, Figure $1 C$ and 1D). We then performed univariate and multivariate Cox regression analyses to determine whether preserved Rab37, low TIMP1 and high PKC $\alpha$ expression pattern is an independent prognostic factor. The univariate Cox regression analysis revealed that patients with preserved Rab37, low TIMP1 and PKC $\alpha$ overexpression, with late stage, larger tumor size status, lymph node metastases or distant organs metastases had an increased risk for poor outcome (Table 2). Remarkably, multivariate Cox regression analysis by including all variables with a significant association in univariate analysis showed that patients with preserved Rab37, low TIMP1 and high PKC $\alpha$ expression pattern correlated with a relative risk of death of $3.81(P=0.002$, confidence interval $=1.66-8.72$; Table 2), suggesting that preserved Rab37, low TIMP1 and high PKC $\alpha$ expression was an independent risk factor of poor outcome. Taken together, these clinical results link PKC $\alpha$ overexpression with poor clinical outcome even in the group of patients with preserved expression of metastatic suppressor Rab37.

\section{Phosphorylation of Rab37 by PKC $\alpha$ abrogates Rab37 GTP binding state}

Little is known about Rab37 phosphorylation in epithelial cells. We thus investigated whether Rab37 was phosphorylated in lung cancer cells by transfecting PC-14 cells with an empty vector (EV) or a plasmid carrying HAtagged Rab37 wild-type (WT) insert. The phosphorylation of Rab37 was detected on the serine/threonine residue(s) (Supplementary Figure 1B). Further, in vitro kinase assay was performed to verify whether PKC $\alpha$ was responsible for phosphorylation of Rab37. As shown in Figure 2, phosphorylation levels of GST-Rab37 was enhanced with increasing PKC $\alpha$ activity (Figure 2A), whereas blocking PKC $\alpha$ activity by Go6976 inhibited the incorporation of $\left[\mathrm{g}^{32} \mathrm{p}\right.$ ] ATP into GST-Rab37 (Figure 2B). These results indicated that PKC $\alpha$ is capable of phosphorylating Rab37 in vitro. 
Table 1: Clinicopathological parameters in lung cancer patients enrolled in the present study

\begin{tabular}{|c|c|c|c|c|c|c|c|}
\hline \multirow{2}{*}{ Characteristics } & \multirow{2}{*}{ Total } & \multicolumn{2}{|c|}{ Rab37 expression } & \multicolumn{2}{|c|}{ TIMP1 expression } & \multicolumn{2}{|c|}{ PKC $\alpha$ expression } \\
\hline & & Low (\%) & Preserved (\%) & Low (\%) & Preserved (\%) & Overexpressed (\%) & Low (\%) \\
\hline & $N=158$ & $51(32.3 \%)$ & $107(67.7 \%)$ & $72(45.6 \%)$ & $86(54.4 \%)$ & $85(53.8 \%)$ & $73(46.2 \%)$ \\
\hline \multicolumn{8}{|l|}{ Type } \\
\hline $\mathrm{SCC}$ & 21 & $9(42.9 \%)$ & $12(57.1 \%)$ & $17(81.0 \%)$ & $4(19.0 \%)$ & $16(76.2 \%)$ & $5(23.8 \%)$ \\
\hline $\mathrm{ADC}$ & 137 & $42(30.7 \%)$ & $95(69.3 \%)$ & $55(40.1 \%)$ & $82(59.9 \%)^{0.001}$ & $69(50.4 \%)$ & $68(49.6 \%)^{0.027}$ \\
\hline \multicolumn{8}{|l|}{ Stage } \\
\hline Stage I-II & 107 & $27(25.2 \%)$ & $80(74.8 \%)$ & $47(43.9 \%)$ & $60(56.1 \%)$ & $58(54.2 \%)$ & $49(45.8 \%)$ \\
\hline Stage III-IV & 51 & $24(47.0 \%)$ & $27(53.0 \%)^{0.009}$ & $25(49.0 \%)$ & $26(51.0 \%)$ & $27(53.0 \%)$ & $24(47.0 \%)$ \\
\hline \multicolumn{8}{|l|}{ T stage } \\
\hline Stage 1-2 & 127 & $38(29.9 \%)$ & $89(70.1 \%)$ & $53(41.7 \%)$ & $74(58.3 \%)$ & $68(53.5 \%)$ & $59(46.5 \%)$ \\
\hline Stage 3-4 & 29 & $12(41.4 \%)$ & $17(58.6 \%)$ & $18(62.1 \%)$ & $11(37.9 \%)^{0.047}$ & $17(58.6 \%)$ & $12(41.4 \%)$ \\
\hline \multicolumn{8}{|l|}{ N stage } \\
\hline N0 & 88 & $23(26.1 \%)$ & $65(73.9 \%)$ & $41(46.6 \%)$ & $47(53.4 \%)$ & $51(58.0 \%)$ & $37(42.0 \%)$ \\
\hline$>\mathrm{N} 1$ & 69 & $27(39.1 \%)$ & $42(60.9 \%)$ & $30(43.5 \%)$ & $39(56.5 \%)$ & $34(49.3 \%)$ & $35(50.7 \%)$ \\
\hline \multicolumn{8}{|l|}{ M stage } \\
\hline M0 & 133 & $38(28.6 \%)$ & $95(71.4 \%)$ & $58(43.6 \%)$ & $75(56.4 \%)$ & $66(49.6 \%)$ & $67(50.4 \%)$ \\
\hline$>\mathrm{M} 1$ & 23 & $12(52.2 \%)$ & $11(47.8 \%)^{0.025}$ & $13(56.5 \%)$ & $10(43.5 \%)$ & $19(82.6 \%)$ & $4(17.4 \%)^{0.003}$ \\
\hline \multicolumn{8}{|l|}{$\begin{array}{l}\text { TIMP1 } \\
\text { expression }\end{array}$} \\
\hline Low & 72 & $38(52.8 \%)$ & $34(47.2 \%)$ & -- & -- & $54(63.5 \%)$ & $18(24.7 \%)$ \\
\hline Preserved & 86 & $13(15.1 \%)$ & $73(84.9 \%)^{0.001}$ & -- & -- & $31(36.5 \%)$ & $55(75.3 \%)^{0.001}$ \\
\hline
\end{tabular}

NOTE: SCC, squamous cell carcinoma; ADC, adenocarcinoma; --, not applicable.

The data was analyzed by Pearson $\chi^{2}$ test. $P$ values with statistical significance are as indicated.

Notably, an increased in vitro $\mathrm{PKC} \alpha$ kinase activity was observed as evident by autophosphorylated signals of $\mathrm{PKC} \alpha$ (Figure 2B). To test whether $\mathrm{PKC} \alpha$ phosphorylates Rab37 in lung cancer cell-based studies, we first detected the PKCa activity by measuring the phospho-substrates of PKC $\alpha$ in cells with overexpression of PKC $\alpha$ (Supplementary Figure 2). Next, we used PMA, a potent activator of PKC $\alpha$, to stimulate Rab37 phosphorylation in PC-14 cells stably expressing WTRab37. PMA induced Rab37 phosphorylation on serine/ threonine (p-S/T) residue(s), and the phosphorylated signal of Rab37 was inhibited by knockdown of PKC $\alpha$ (Figure 2C). Interestingly, PMA treatment abrogated Rab37 GTP binding as analyzed by immunoprecipitation (IP) with GTP-Sepharose affinity beads followed by immunoblotting analysis (Figure 2D). Notably, PKC $\alpha$ knockdown dramatically restored Rab37 GTP binding upon PMA treatment, suggesting that PKC $\alpha$-mediated phosphorylation dampened the GTP-bound active state of Rab37 (Figure 2E). Taken together, these results suggested that PKC $\alpha$-mediated Rab37 phosphorylation leads to reduction in GTP-bound Rab37.

\section{PKCa abolishes Rab37-mediated exocytosis function and metastasis suppression activity}

To further characterize the effects of PKC $\alpha$ mediated Rab37 phosphorylation on Rab37-regulated cargo secretion, we measured the amounts of Rab37mediated secretion of cargos, TIMP1 and TSP1, in the conditioned medium (CM) from WT-Rab37-stable PC-14 cell line with transient PKC $\alpha$-overexpression or knockdown. Notably, lower amounts of TIMP1 and TSP1 were observed in the $\mathrm{CM}$ from PKC $\alpha$ overexpressing cells (Figure 3A), while knockdown of PKC $\alpha$ increased TIMP1 and TSP1 level in CM (Figure 3B). These data were consistent with the model predicting the suppression of Rab37-mediated cargo secretion by PKC $\alpha$. To further determine the mechanism for the suppression of Rab37mediated cargo secretion by PKC $\alpha$, FLAG-tagged WTRab37-specific vesicles were isolated and analyzed for levels of TIMP1 and TSP1. The results showed that TIMP1 and TSP1 were associated with Rab37-containing vesicle fractions and the association was abrogated by overexpressing PKC $\alpha$ (Figure 3C). In contrast, 
Table 2: Cox regression analysis of risk factors for cancer-related death in lung cancer patients

\begin{tabular}{|c|c|c|c|c|}
\hline \multirow{2}{*}{ Characteristics } & \multicolumn{2}{|c|}{ Univariate analysis } & \multicolumn{2}{|c|}{ Multivariate analysis } \\
\hline & HR $(95 \%$ CI $)$ & $P$-value ${ }^{\text {a }}$ & HR $(95 \%$ CI $)$ & $P$-value ${ }^{a}$ \\
\hline \multicolumn{5}{|l|}{$\begin{array}{l}\text { Rab37/TIMP1/PKCa } \\
\text { expression }\end{array}$} \\
\hline$+/+/-$ & 1.00 & & 1.00 & \\
\hline Others & $1.27(0.68-2.38)$ & 0.450 & $1.53(0.78-3.01)$ & 0.215 \\
\hline$+/-/+$ & $3.15(1.55-6.38)$ & 0.001 & $3.81(1.66-8.72)$ & 0.002 \\
\hline \multicolumn{5}{|l|}{ Type } \\
\hline $\mathrm{ADC}$ & 1.00 & & $--^{b}$ & \\
\hline $\mathrm{SCC}$ & $1.24(0.35-1.41)$ & 0.283 & $--^{b}$ & $--^{b}$ \\
\hline \multicolumn{5}{|l|}{ Stage } \\
\hline Stage I-II & 1.00 & & 1.00 & \\
\hline Stage III-IV & $1.73(1.05-2.87)$ & 0.032 & $1.28(0.64-2.57)$ & 0.488 \\
\hline \multicolumn{5}{|l|}{ T stage } \\
\hline Stage $1-2$ & 1.00 & & 1.00 & \\
\hline Stage 3-4 & $1.89(1.08-3.31)$ & 0.026 & $1.07(0.54-2.11)$ & 0.848 \\
\hline \multicolumn{5}{|l|}{ N stage } \\
\hline N0 & 1.00 & & 1.00 & \\
\hline$>\mathrm{N} 1$ & $1.83(1.09-3.07)$ & 0.022 & $1.75(0.87-3.49)$ & 0.115 \\
\hline \multicolumn{5}{|l|}{ M stage } \\
\hline M0 & 1.00 & & 1.00 & \\
\hline$>$ wM1 & $1.83(1.02-3.29)$ & 0.042 & $1.01(0.52-1.95)$ & 0.987 \\
\hline
\end{tabular}

NOTE: ADC, adenocarcinoma; SCC, squamous cell carcinoma; CI, confidence interval; HR, hazard ratio.

${ }^{a}$ Bold values represented statistical significance $(P<0.05)$.

${ }^{b}$ The variables without significant HR in univariate analysis were not included in the multivariate analysis.

siRNA-knockdown of PKC $\alpha$ expression increased the association of TIMP1 and TSP1 with Rab37 in the same vesicle fractions (Figure 3D).

Furthermore, we performed immuno-electron microscopy (immuno-EM) assay for ultrastructural localization of Rab37 and TIMP1 and found that Rab37 and TIMP1 localized on the same vesicles (47\%, upper; Figure 3E and Supplementary Figure 3A), while there was a marked reduction of colocalization of Rab37 and TIMP1 in PKC $\alpha$-overexpressing cells where most Rab37 proteins were dissociated from the vesicle membranes and scattered with cytoplasm (18\%, middle; Figure $3 \mathrm{E}$ and Supplementary Figure 3A). The reduction in Rab37 and TIMP1 colocalization was recovered in cells with $\mathrm{PKC} \alpha$ knockdown (52\%, lower; Figure 3E and Supplementary Figure 3A). Notably, the fractionation assay showed that WT-Rab37, but not phospho-deficient alanine substitution mutant at threonine-172 (T172A), associated with less membrane fraction upon activation of PKC $\alpha$ by PMA treatment (Supplementary Figure 3B). Additional mass spectrometry analysis and site-direct mutagenesis experiments to identify the Rab37 phosphorylation are described below.
Finally, the regulation of TIMP1 trafficking dynamics by Rab37 was analyzed by using total internal reflection fluorescence (TIRF) microscopy, which allowed us to monitor real-time images of fluorescently labeled vesicles located in close proximity to the plasma membrane (upper, Figure 3F; Supplementary Movie 1). Overexpression of PKC $\alpha$ dramatically attenuated Rab37mediated TIMP1 exocytotic transport in WT-Rab37 cells (middle, Figure 3F; Supplementary Movie 2), whereas the TIMP1-containing vesicles trafficking events were more in PKC $\alpha$-silenced WT-Rab37 cells than that in control WT-Rab37 cells (lower; Figure 3F; Supplementary Movie 3). These data therefore supported a mechanism by which PKC $\alpha$ blocks Rab37-mediated cargos secretion by facilitating the dissociation of phosphorylated Rab37 from its targeting vesicles.

\section{PKC $\alpha$ phosphorylates Rab37 on threonine-172}

To identify the phosphorylation site(s) on Rab37 by $\mathrm{PKC} \alpha$, an in vitro kinase assay with recombinant PKC $\alpha$ and purified GST-Rab37 was performed. Mass spectrometry analysis of the phosphorylated Rab37 after 
in vitro $\mathrm{PKC} \alpha$ kinase reaction identified six potential sites on Rab37, designated as S94, S149, T160, T172, S203 and S212 that may be phosphorylated by PKC $\alpha$. The phosphomimetic aspartate (D) substitution mutants of these six candidate sites were constructed. Since Rab37 acts as a cell motility suppressor, the effects of these mutants on cell motility were then determined by transwell migration and invasion assays. Among the mutants, only the replacement of threonine-172 with aspartate T172D showed significant promotion of cell migration and invasion (Figure 4A). We also performed cell migration and invasion assays using Rab37 mutant constructs with the six potential residues mutated to alanine (A). These phospho-deficient mutants displayed similar migration and invasion abilities compared with WT-Rab37 (Supplementary Figure 4). These data suggested that PKC $\alpha$ may phosphorylate
Rab37 at T172 to alter the Rab37-mediated functions. We then performed in vitro kinase assay to further confirm $\mathrm{PKC} \alpha$ phosphorylation of Rab37 at T172. The p-S/T signal disappeared when GST-Rab37-T172A was used as the substrate of PKC $\alpha$ or in the presence of PKC $\alpha$ inhibitor Go6976 (Figure 4B). Moreover, PMA-induced Rab37 phosphorylation was inhibited in Rab37-T172Aexpressing PC-14 cells (Figure 4C). These in vitro and cell-based results indicated that T172 of Rab37 is phosphorylated by PKC $\alpha$.

\section{Threonine-172 phosphorylation disrupts Rab37 GTP binding}

Since PKC $\alpha$-mediated Rab37 phosphorylation reduced Rab37 in GTP-bound form (Figure 2) and
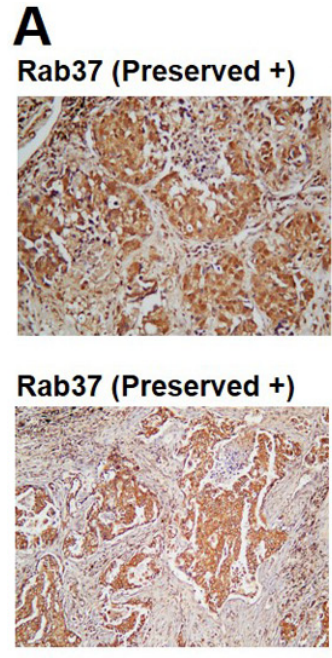

TIMP1 (Low - )

C

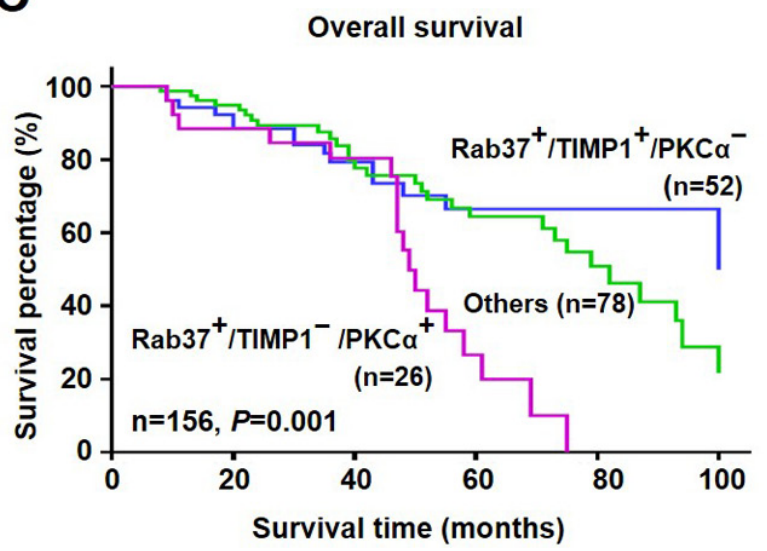

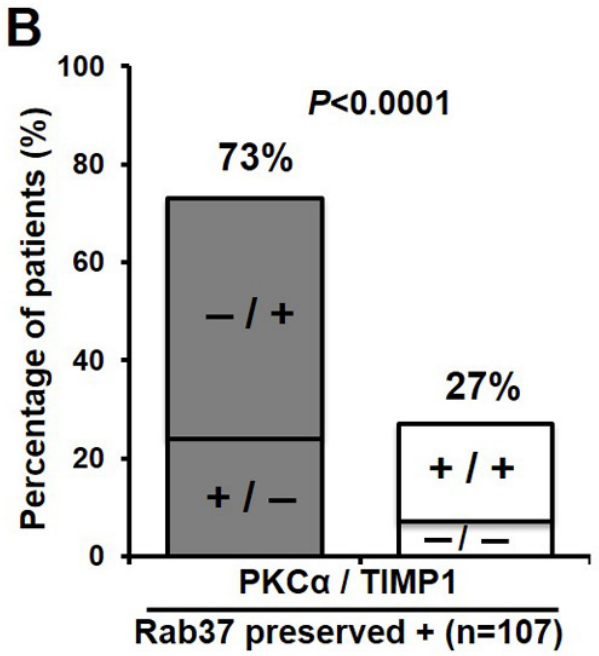

Disease-free survival

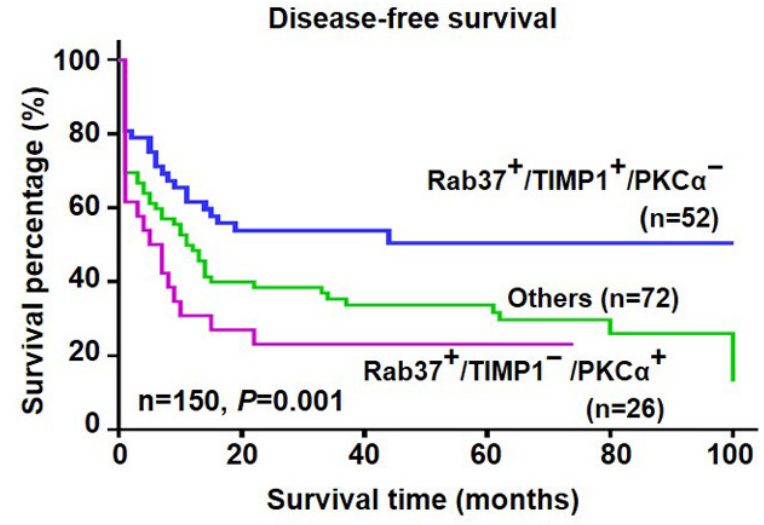

Figure 1: High level of PKCa associated with poor survival in the lung cancer patients with preserved expression of Rab37 and low level of TIMP1. (A) Immunohistochemistry analysis of Rab37, TIMP1 and PKC $\alpha$ expression in lung specimen from two lung cancer patients. (B) Concordance analysis of TIMP1 (+, preserved; -, low expression) and PKC $\alpha(+$, overexpression; -, low expression) in lung cancer patients with preserved Rab37 expression $(n=107)$. The percentage of discordant group (gray bar) and concordant group (white bar) was indicated in the plot. $P$-value was calculated by Pearson $\chi^{2}$-test. (C and D). Overall survival and disease-free survival of Kaplan-Meier analysis were shown. The normal expression of Rab37 and TIMP1, overexpressed PKC $\alpha$ level were designated as "+"; and low expression of indicated proteins as "-". $P$-values were determined by log-rank test. Lung cancer patients with expression profile of normal Rab37, low TIMP1 and overexpressed PKC $\alpha$ showed worse overall survival (C) and disease-free survival (D). 
impaired Rab37-mediated exocytic function (Figure 3), we therefore speculated whether PKC $\alpha$-mediated T172 phosphorylation of Rab37 inhibited GTP binding ability of Rab37. As shown in Figure 4D, GTP-bound active glutamine-to-leucine of Rab37 (Q89L-Rab37) mutant displayed a significant increase in GTP binding compared to WT-Rab37. Interestingly, both T172D-Rab37 mutant and Q89L/T172D-Rab37 double mutant reduced the level of GTP-bound active Rab37 (Figure 4D), suggesting that T172 phosphorylation inhibited the activity of Rab37 via reducing the abundance of GTP-bound Rab37. Moreover, the amount of GTP-bound Rab37 in WT-Rab37 cells was diminished upon PMA treatment, whereas the GTPbound form of phospho-deficient mutant T172A was not affected by PMA stimulation (Figure 4E, lanes 5 and 6). Collectively, our results suggested that PKC $\alpha$-mediated Rab37 phosphorylation at T172 attenuates Rab37 activity through reduction in GTP-bound state.
Phosphorylation of Rab37 on threonine-172 by PKC $\alpha$ abolishes its colocalization with TIMP1, resulting in loss of its motility suppressive function

Since PKC $\alpha$-mediated phosphorylation disrupted exocytic trafficking of TIMP1 and TSP1 by Rab37 (Figure 3) and reduced the GTP-bound state and membrane bound form of Rab37 (Figures 2 and 4), we further interrogated the cellular localization of Rab37 and its cargo TIMP1 in Rab37-WT, Rab37-T172D and Rab37T172A cells. Indeed, ectopically expressed Rab37-WT or Rab37-T172A co-localized with TIMP1, whereas Rab37T172D displayed distinct cellular distribution from TIMP1 in PC-14 cells (Figure 5A, merged panel). Accordingly, $\mathrm{PKC} \alpha$ expression markedly decreased CM concentration of TIMP1 and TSP1 in Rab37-WT cells, but not in Rab37T172A cells (Figure 5B). In contrast, lower CM level
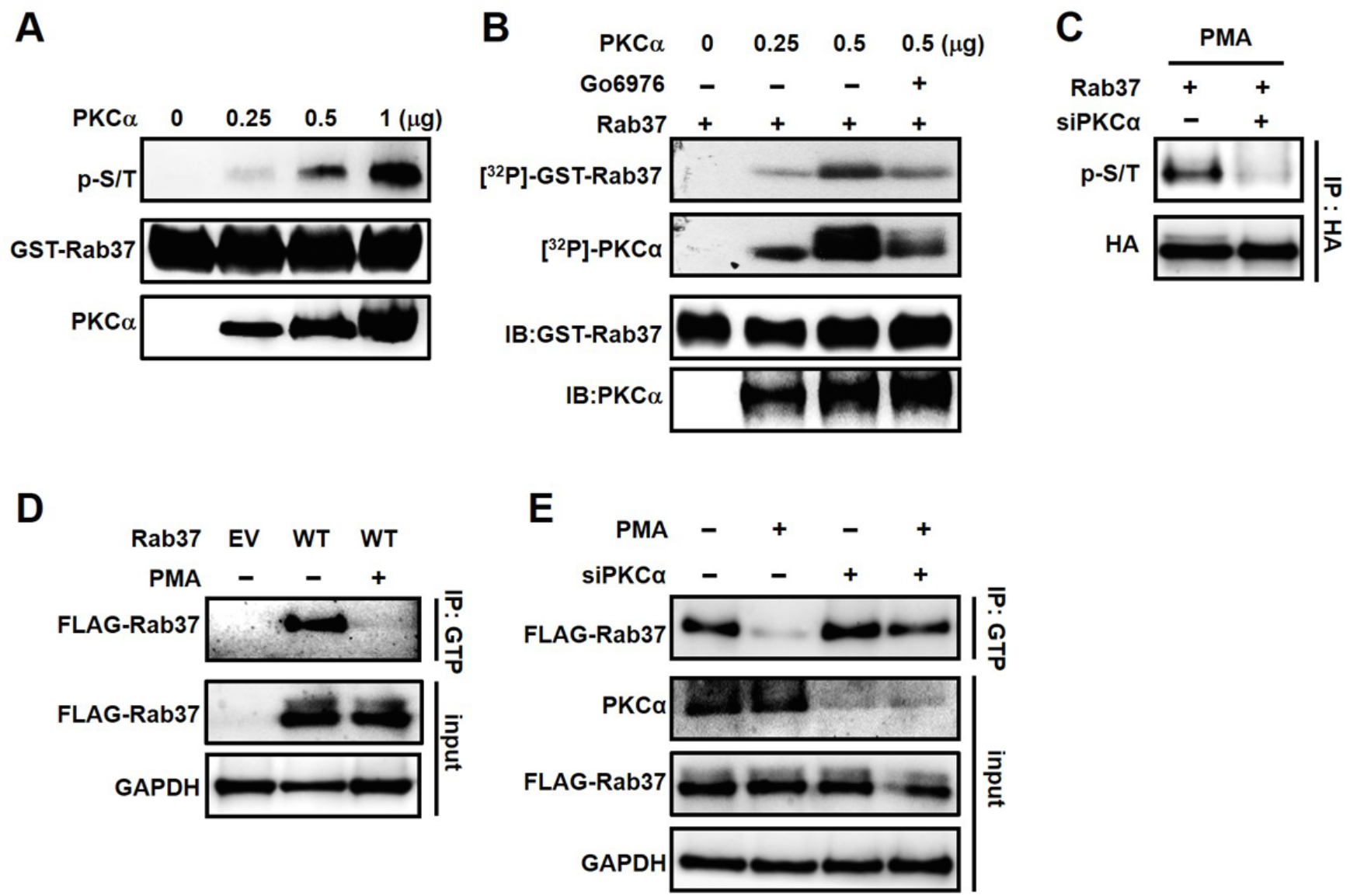

Figure 2: Rab37 phosphorylation by PKCa inhibited GTP binding ability. (A) In vitro kinase assay was performed by incubating recombinant PKC $\alpha$ with purified GST-Rab37. The resulting mixtures were subjected to immunoblotting with antibodies against p-S/T proteins, Rab37 and PKC $\alpha$. (B) Purified GST-Rab37 was incubated with $\left[\gamma_{-}{ }^{32} \mathrm{P}\right]$ ATP and recombinant PKC $\alpha$ in the presence or absence of PKC $\alpha$ inhibitor Go6976. The autophosphorylation signals of PKC $\alpha$ served as a positive control in our in vitro kinase assay. (C) HA-Rab37 expressing PC-14 cells were transfected with non-specific siRNA (designated "-") or siRNA mix against PKCa (designated “+”) for 48 hours. After treatment of PMA for 30 minutes, the cell lysates were prepared for IP with anti-HA antibody and followed by immunoblotting analysis with anti-p-S/T and -HA antibodies. (D and E) The treatment of PMA(D) and siRNA targeting PKC $\alpha$ (E) were the same as (C). The cell lysates were IP with GTP-conjugated beads followed by immunoblotting analysis with indicated antibodies. 

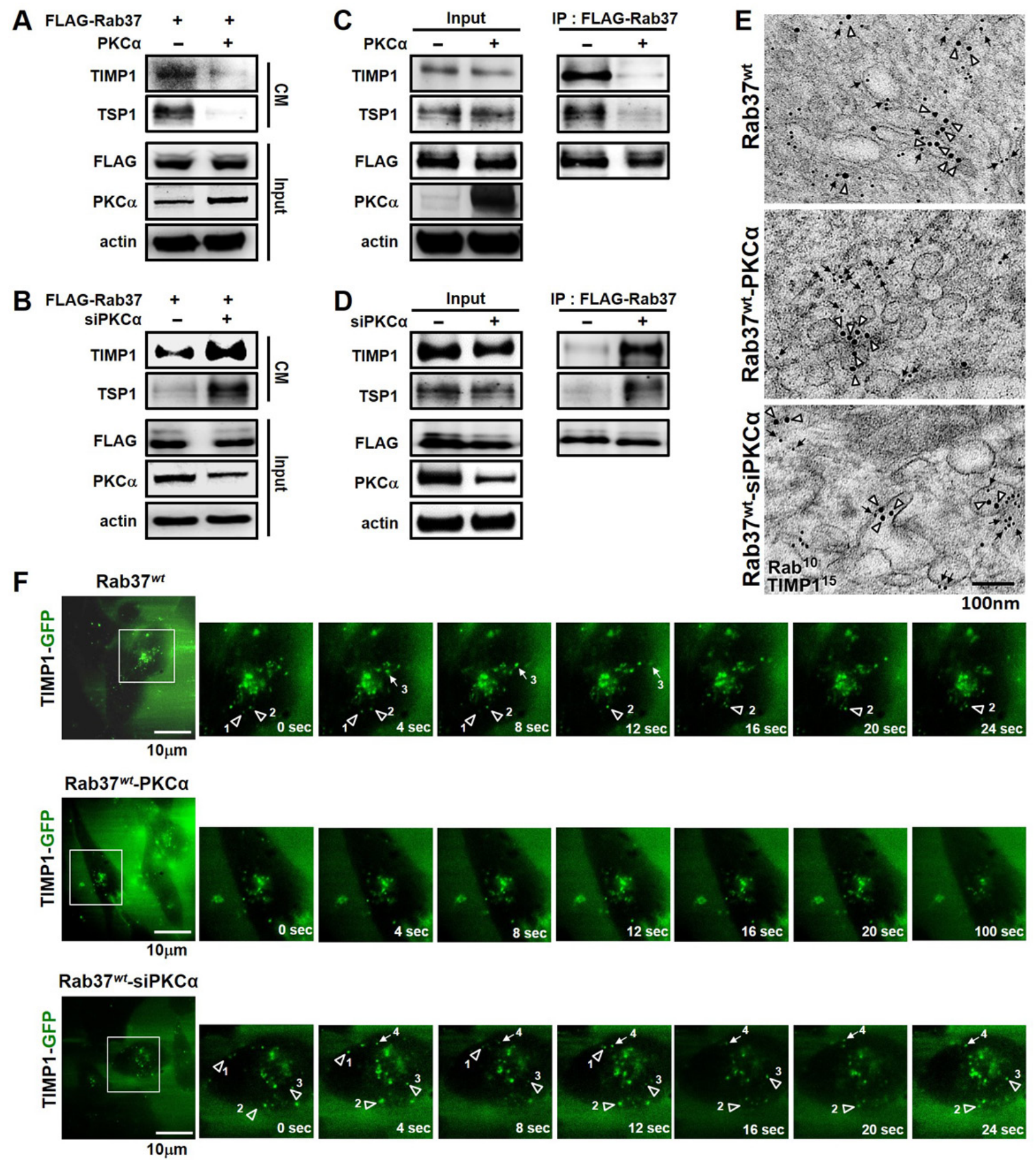

Figure 3: PKC $\alpha$ expression impaired Rab37-mediated exocytotic transport of TIMP1 and TSP1. (A) PC-14 cells stably expressing FLAG-tagged Rab37 (FLAG-Rab37) were transfected with empty or PKCa-expressing vectors. The cell conditioned medium (CM) was harvested and subjected to immunoblotting. (B) siRNA against PKC $\alpha$ was introduced into cells stably expressing FLAG-Rab37, the TIMP1 and TSP1 levels in CM was analyzed by immunoblotting. (C and D) Cells stably expressing FLAG-Rab37 were transfected with expressing vector (C) or siRNA oligos of PKC $\alpha$ (D). Vesicles were isolated by centrifugations and subjected to IP with anti-FLAG antibody followed by blotting for FLAG-Rab37 and endogenous TIMP1 and TSP1. (E) Cells stably expressing FLAG-Rab37wt were transfected with empty (upper) or PKC $\alpha$-expressing vector (middle) or siRNA against PKC $\alpha$ (lower). The co-localization of TIMP1 (15 nm of gold, triangle) and Rab37 (10 nm of gold, arrow) in vesicles was observed in immuno-EM images. Scale bars $100 \mathrm{~nm}$. (F) Selected frames from time-lapse movies of TIMP1 trafficking in cells expressing FLAG-Rab37wt, and GFP-TIMP1 together with overexpression (Rab37wt$\mathrm{PKC} \alpha$ ) or knockdown (Rab37wt-siPKC $\alpha$ ) of PKC $\alpha$ were shown. The numbers labeled horizontal (arrow) or vertical (triangle) movement of GFP-TIMP1. Enlarged images of the boxed areas from time-lapse movies with time intervals in seconds shown. Scale bars $10 \mu \mathrm{m}$. 
of TIMP1 and TSP1 were observed in cells expressing T172D mutant. Note that siRNA-mediated knockdown of $\mathrm{PKC} \alpha$ significantly increased the CM concentration of TIMP1 and TSP1 in Rab37-WT cells. In addition, change in PKC $\alpha$ level did not alter the exocytic trafficking activity in the Rab37-T172D mutant-expressing cells (Figure 5C).
Furthermore, overexpression of $\mathrm{PKC} \alpha$ could not further interfere with the regulation on cell motility in the T172A cells (Figure 5D). Accordingly, silencing PKC $\alpha$ also could not lead to additional enhancement of cell migration in the T172D-expressing cells (Figure 5E). Of note, the amount of secreted TIMP1 and TSP1 level in CM from

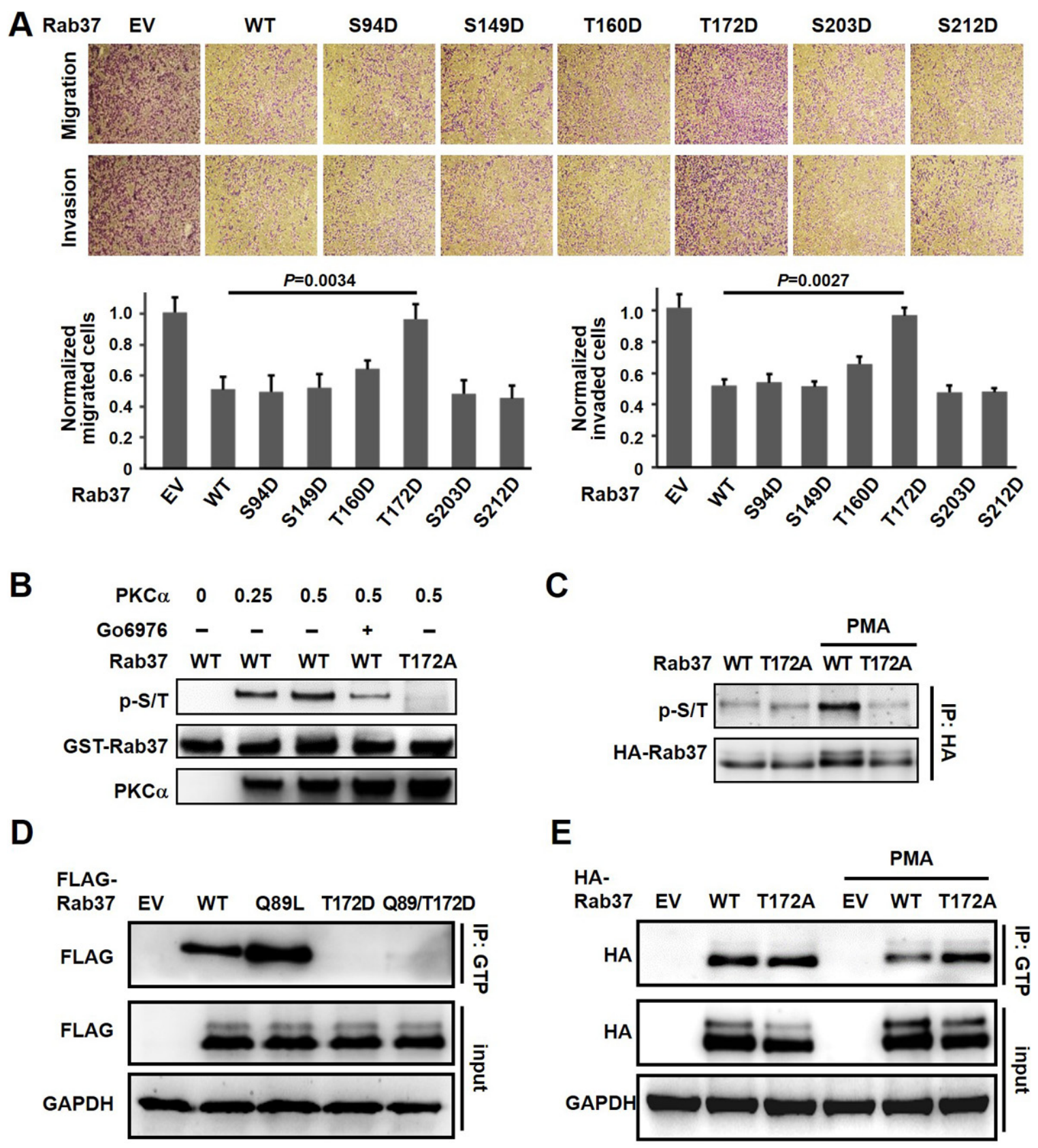

Figure 4: PKCa phosphorylated Rab37 at T172 and inhibited GTP binging ability. (A) PC-14 cells were transfected with various Rab37 phospho-mimetic D mutants followed by analysis of cell migration and invasion abilities (upper). The quantitative results were determined by two-tailed Student's $t$-test (lower). Data represented mean \pm SD. $(n=3)$. Original magnification: $100 X$. (B) Recombinant PKC $\alpha$ and purified WT or GST-tagged Rab37-T172A were used in an in vitro kinase assay in the presence or absence of Go6976. The phosphorylation signal of Rab37 was analyzed by immunoblotting. (C) PC-14 cells expressing HA-tagged WT or T172A mutant of Rab37 protein were treated with PMA. The lysates were collected for IP with anti-HA antibody and immunocomplexes were blotted for $\mathrm{p}$-S/T proteins and HA-tagged Rab37. (D) Lysates from cell expressing various FLAG-tagged Rab37 mutants were subjected to GTP binding assay. (E). EV, WT or T172A mutant of HA-tagged Rab37 were transfected into PC-14 cells, the lysates were used for GTP binding assay following PMA treatment. 
Rab-T172A and -T172D cells were consistent with the scenario that Rab37 phosphorylation at T172 attenuated cargo secretion and thus impaired Rab37-mediated suppression of cell migration and invasion (Figure 5). Similar results of inhibitory effects of PKC $\alpha$-mediated T172 phosphorylation of Rab37 on cellular distribution, Rab37-mediated TIMP1 and TSP1 secretion and motility suppression were observed in another lung cancer cell line A549 (Supplementary Figure 5). These results indicated that PKC $\alpha$-mediated $\mathrm{T} 172$ phosphorylation of Rab37 eliminates TIMP1 secretion and thus promotes cell motility.

\section{Phospho-mimetic mutant of Rab37 on threonine-172 promotes metastasis in vivo}

The inhibition of Rab37 activity by PKC $\alpha$-mediated phosphorylation in cell-based model prompted us to investigate whether T172 phosphorylation of Rab37 impaired metastasis suppression in animals. We injected
EV control, WT- and T172D-Rab37 expressing cells into the tail vein of nude mice. Consistent with our findings in cell model, T172D-Rab37 cells markedly enhanced lung metastases in injected mice compared with mice injected with WT-Rab37 cells (Figure 6A-6C). Importantly, when the concordant expression of WT-Rab37 and TIMP1 was confirmed in vivo, T172D-derived lung metastases showed similarly high Rab37 expression but lower TIMP1 staining as compared to that in WT-Rab37-derived lung metastases (Figure 6D). These in vivo results further support the notion that Rab37 phosphorylation at T172 inactivates the Rab37-mediated TIMP1 secretion and thus promotes tumor metastases.

\section{DISCUSSION}

In this study, we identify a novel mechanism for repression of $\mathrm{Rab37}$ activity by $\mathrm{PKC} \alpha$-mediated phosphorylation via interfering with its GTP binding state. We unravel a phosphorylation site on Rab37 at
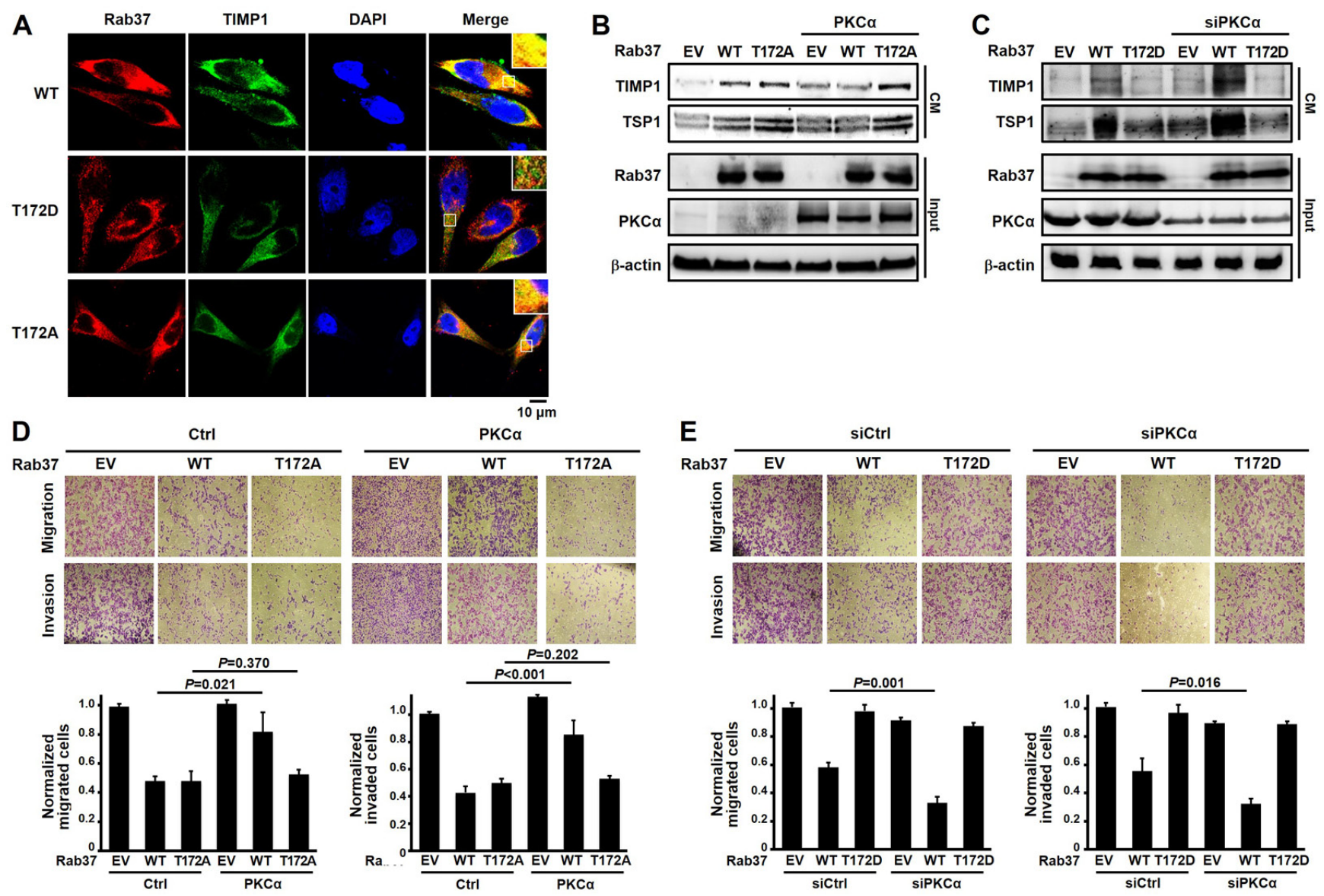

Figure 5: PKCa-mediated T172 phosphorylation of Rab37 was critical for modulating Rab37 activity. (A) Confocal microscopic images of Rab37 (red), TIMP1 (green) and nucleus staining (blue) in WT-, T172D- and T172A-Rab37 expressing PC-14 cells. Enlarged images shown in insets of the merge panel. Scale bars, $10 \mu \mathrm{m}$. (B and C) CM were collected from PKC $\alpha$-overexpressing (B) or -silencing (C) cells, which expressed T172A- or T172D-Rab37, respectively. The TIMP1 and TSP1 levels were analyzed by immunoblotting. (D and E) the manipulation of PKC $\alpha$ and mutant Rab37 in cells used in (B and C) were subjected to cell migration and invasion assay (upper) and quantitative analyses (lower). Data represented mean $\pm \mathrm{SD}$. $(n=3)$. Original magnification: $100 \mathrm{X}$. 
threonine-172, which is critical for regulation of Rab37 activities including GTP-bound conformation, exocytosis of the cell motility inhibitors TIMP1 and TSP1, and metastasis suppression in vitro, in vivo and clinically (Figure 7). From a clinical point of view, we identified a group of lung cancer patients with a profile of high
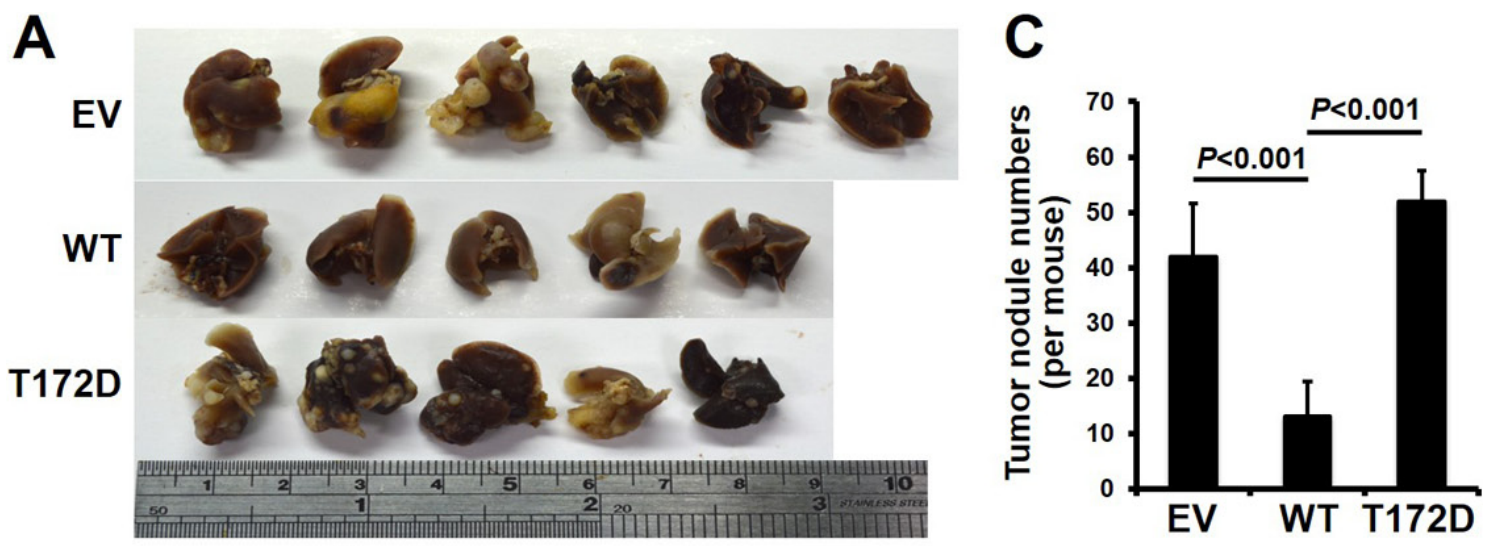

B
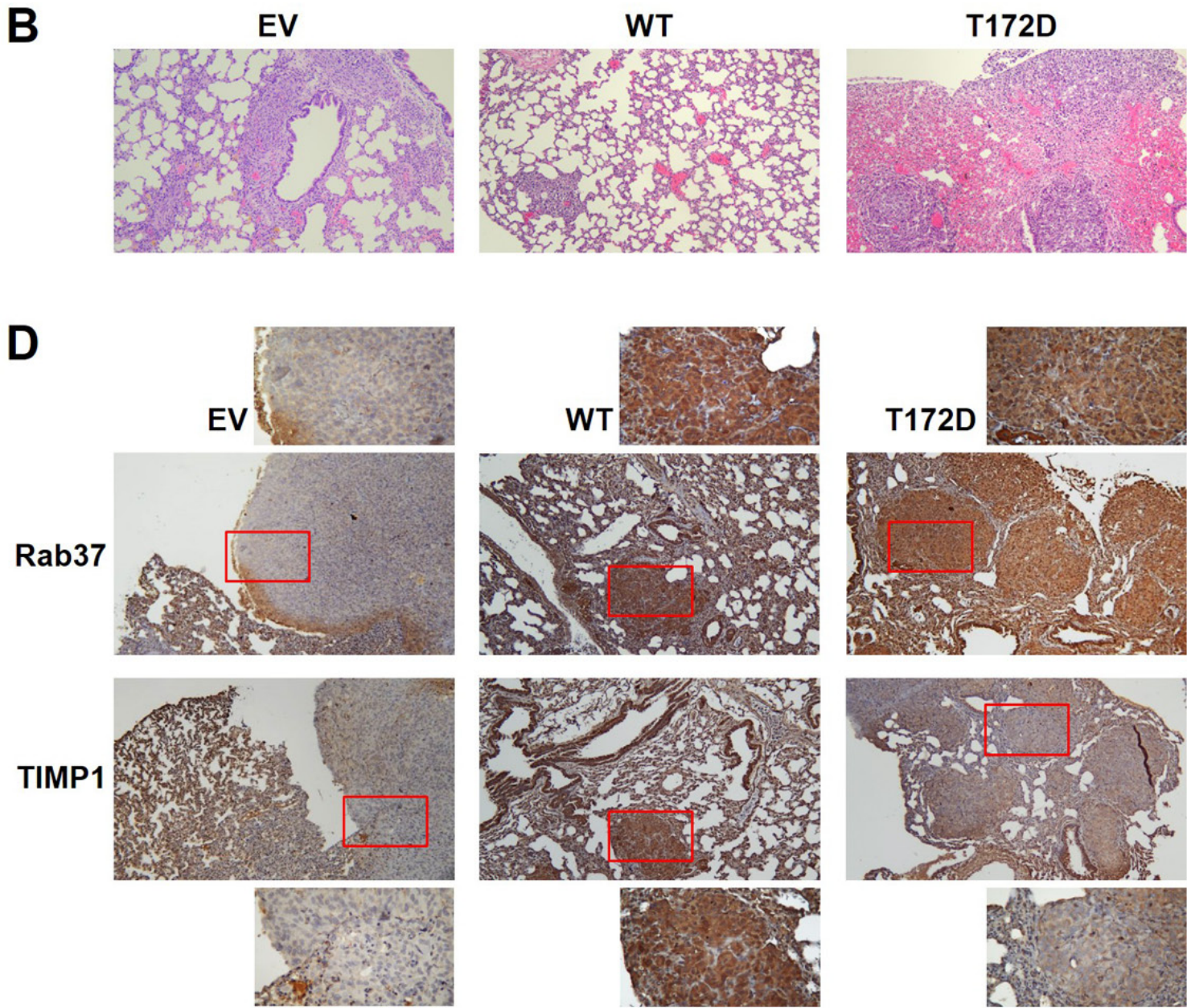

Figure 6: Phospho-mimetic T172D mutant of Rab37 lost its metastasis suppression ability in vivo. (A) The lung images from nude mice 6 weeks after injection of PC-14 cells stably expressing EV-, WT- or T172D-Rab37 via the tail vein. (B) and (C) H\&E staining shows more lung colonization and bigger tumor mass in EV control and T172D groups compared with WT group (B) (Original magnification: $200 \times)$. Numbers of tumor nodules are shown in the histogram plot (C). $P$-values were calculated by two-tailed Student's $t$-test. Data represented mean \pm SD. ( $n=5-6$ mice/group). (D) Lung tissues from EV-, WT- or T172D-injected nude mice were collected 6 weeks post-injection and subjected to immunohistochemistry to examine the expression of Rab37 and TIMP1. Enlarged images of insets are shown (Original magnification: 200×). 
PKC $\alpha$, high Rab37 and low TIMP1 secretion, and this group of patients displayed poor prognosis (Figure 1 and Tables 1-2). The mechanism for phosphorylation of Rab37 by PKC $\alpha$ leading to malfunction of Rab37mediated exocytosis of TIMP1 and aggressive metastasis is further supported by our cell and animal data (Figures 3-6). Our findings provide the molecular insight into how Rab37 activity is regulated by phosphorylation coupled to the GTP/GDP binding mechanism (Figures 2 and 4) with prognostic implications.

Several Rab GTPases are identified as targets for PKC isozymes such as Rab5a, Rab6 and Rab11 [20-22]. Phosphorylation of Rab5a by PKCe promotes Rac1 activation to facilitate actin remodeling [20]. Conventional PKC-mediated Rab11 and Rab6 phosphorylation results in impaired endosomal recycling and redistribution in cytosolic fraction, respectively [21, 22]. However, it remains unclear whether PKC-mediated phosphorylation regulates activities in vesicle trafficking and cargo transport of these Rabs. Our results demonstrated that phosphorylation of Rab37 by PKC $\alpha$ suppressed the exocytotic activity of cargos, TIMP1 and TSP1, mediated by Rab37 (Figures 3-5). Images from confocal microscopy and immuno-EM analysis illustrated dislocation of Rab37 from TIMP1-containing vesicles upon $\mathrm{PKC} \alpha$ overexpression (Figure 5A and Figures 3A-3E). Importantly, this dislocation may result from reduction of active GTP-bound Rab37 (Figures 2D and E). In addition, our results indicated that T172 phosphorylation inhibited GTP-bound Rab37 state (Figure 4E), suggesting that T172 phosphorylation has a crucial role in regulating the GTPbound Rab37 activity.
Interestingly, threonine-172 is located in the G5 box (ETSAK), a conserved region that is involved in the guanine-nucleotide binding (Supplementary Figure 6). This raises the possibility that $\mathrm{T} 172$ phosphorylation impairs GTP binding via electric repulsion or interference of GTP entry into the binding pocket of Rab37 spatiotemporally. In addition, Rab GTPase activity is determined by interaction with effector complex and GDP/GTP binding state, which is catalyzed by GTPaseactivating proteins (GAP) and guanine nucleotide exchange factors (GEF) [23]. A glutamine-to-leucine mutation of Rab37 (Q89L) stabilizes Rab in an active conformation by prevention of GAP-mediated GTP hydrolysis [24]. Unexpectedly, overexpression of PKC $\alpha$ abrogated the suppression of Q89L-Rab37 active mutant on cell motility (Supplementary Figure 7) and T172 phosphorylation of Rab37 reduced the level of GTPbound Q89L mutant (Figure 4D). We therefore speculated that T172 phosphorylation of Rab37 might affect the interaction with its cognate effectors. Indeed, studies have shown that phosphorylation of Rab4 by $\mathrm{p} 34^{\mathrm{cdc} 2}$ prevents the association of Rab4 with endosomal membrane by dissociating the binding to membrane effector during the cell cycle $[25,26]$. Rab8A phosphorylation on S111 is also observed to impair its binding to Rabin8, the GEF for Rab8A that triggers GDP exchange [27]. It will be interesting to identify the effector proteins and GEF for Rab37 to clarify this notion.

The downstream targets of PKC family as predicted by computational strategies commonly contain linear consensus motifs such as $(\mathrm{S} / \mathrm{T}) \mathrm{X}(\mathrm{K} / \mathrm{R}),(\mathrm{K} / \mathrm{R}) \mathrm{XX}(\mathrm{S} / \mathrm{T})$ or

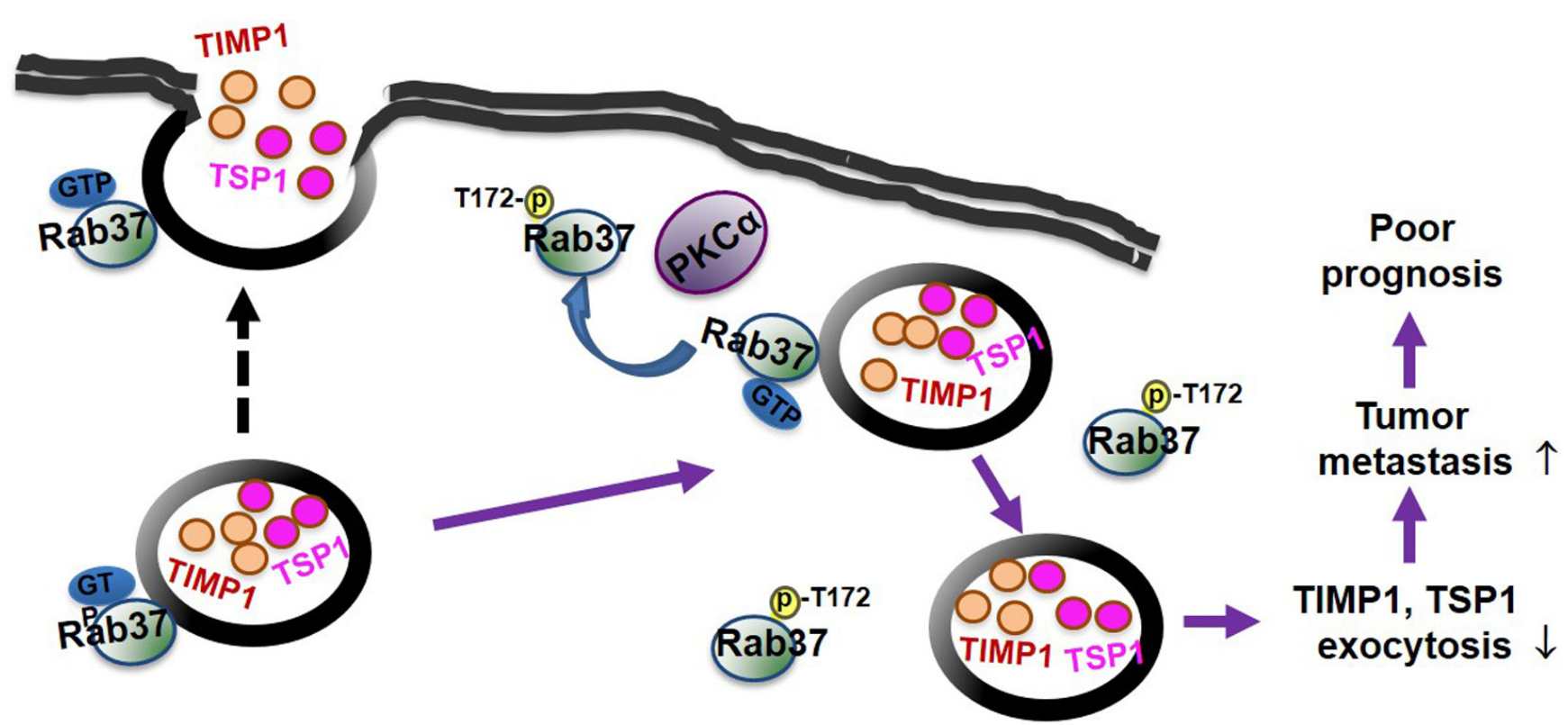

Figure 7: A schematic diagram illustrates that PKCo-mediated Rab37 phosphorylation inhibits the GTP-binding and exocytosis function as well as the metastasis suppressive effects of Rab37 leading to tumor progression and poor prognosis of lung cancer patients. 
$(\mathrm{L} / \mathrm{R}) \mathrm{X}(\mathrm{S} / \mathrm{T})$. Here we performed in vitro kinase assay with recombinant $\mathrm{PKC} \alpha$ and purified Rab37 in conjunction with mass spectrometry analysis and site-direct mutagenesis assay to identify the T172 residue of Rab37 as the site that is phosphorylated by PKC $\alpha$ (Figures 2 and 4). However, T172 is not within a part of linear PKC consensus sequences. Notably, new strategies with advanced chemical biology, mass spectrometry and proteomics have revealed that many protein kinase substrates are phosphorylated on residues that are not within the software-predicted linear consensus motifs [28-30]. Instead, the phosphorylation sites recognized by kinases occur within a region that is created by structure-based non-linear consensus motif [31]. Thus, it is speculated that the sequence surrounding T172 on Rab37 may create a structure-based consensus motif. Further structure biology studies will clarify how PKC $\alpha$ recognizes sequence flanking T172 residue on Rab37.

PKC $\alpha$ has long been recognized as an oncoprotein in several types of cancer by regulating tumor growth and progression [32-36]. Both of our cell-based and animal studies revealed that phospho-mimetic mutant of Rab37 at T172, the substrate site of PKCa, profoundly enhanced cell motility and lung metastasis (Figures 4-6). These results link the oncogenic signaling of PKC $\alpha$ to attenuation of metastasis suppressive function of Rab37.

In conclusion, our study provides a new mechanistic insight into the regulation of Rab37 activity by PKC $\alpha$ dependent phosphorylation leading to inhibition of Rab37-mediated exocytosis of anti-migratory factor/cargo and thus facilitating cell motility and cancer metastasis. Phosphorylation on T172 of Rab37 significantly suppresses its binding affinity for GTP. New diagnostic and therapeutic strategies based on detection of Rab37 phosphorylation and inhibitors against phosphorylated Rab37 could improve the survival in lung cancer patients.

\section{MATERIALS AND METHODS}

\section{Cell culture and reagents}

Human lung cancer cell line PC-14 was obtained from Dr. Pan-Chyr Yang (Department of Internal Medicine, Medical College, National Taiwan University, Taipei, Taiwan). A549 was purchased from ATCC. Stable expression of FLAG-tagged WT-, active Q89L- and inactive T43N-Rab37 PC-14 cells were generated as described previously [18]. The small interfering RNAs pool containing 5'-UAAGGAACCACAAGCAGUA-3', 5'-UUAUAGGGAUCUGAAGUUA-3', 5'-GAAGGGUU CUCGUAUGUCA-3', 5'-UCACUGCUCUAUGGACU UA-3' (Dharmacon, DE, USA) was used to knockdown PKC $\alpha$. Phorbol 12-myristate 13-acetate (PMA) and conventional PKC inhibitor (Go6976) were from SigmaAldrich (St. Louis, MO, USA).

\section{Site-directed mutagenesis and in vitro kinase assay}

Phospho-deficient and -mimetic mutants of Rab37 were generated by PCR-based site-directed mutagenesis. Serine $(\mathrm{S})$ or threonine $(\mathrm{T})$ was mutated to alanine $(\mathrm{A})$ or to aspartate (D). The primer sequences used and PCR reactions are listed in Supplementary Table 1. All constructs were verified by sequencing. The resulting products were then transformed into E.coli. To analyze protein phosphorylation level, the GST-fused Rab37 proteins (Rab37-WT or Rab37-T172A) and recombinant PKC $\alpha$ (Millipore, MA, USA) were incubated with $\left[\gamma^{-32} \mathrm{P}\right]$ ATP followed by autoradiography or by Western blot using antibody against $\mathrm{p}-\mathrm{S} / \mathrm{T}$ peptides (Supplementary Table 2).

\section{Vesicle isolation, immunoprecipitation and cellular fractionation}

Vesicle isolation protocol was modified from Hendrix's report [15]. In brief, cells $\left(2 \times 10^{8}\right)$ were sonicated and supernatants were obtained by centrifugation $\left(3,000 \mathrm{~g}\right.$ for $10 \mathrm{~min}$ at $\left.4^{\circ} \mathrm{C}\right)$ and vesicles were enriched from supernatants by high speed centrifugation $(30,000$ $\mathrm{g}$ for $60 \mathrm{~min}$ at $4^{\circ} \mathrm{C}$ ) using a $40-\mathrm{Ti}$ rotor (Beckman Coulter, CA, USA). The vesicles-containing solution $(800 \mu \mathrm{g})$ was incubated with anti-Flag antibody to isolate Rab37-specific vesicles and the cargos in vesicles were analyzed by immunoblotting using indicated antibodies (Supplementary Table 2). For cellular fractionation, intact cells suspended in the lysis buffer ( $25 \mathrm{mM}$ Hepes, pH6.5, $250 \mathrm{mM}$ sucrose, 1mM EDTA and protease inhibitor cocktail) were pelleted by centrifugation $(250 \mathrm{~g}$ for 10 min). The cytoplasmic (supernatant) and membrane (pellet) fractions were then obtained by centrifugation at $16000 \mathrm{~g}$ for $60 \mathrm{~min}$ at $4^{\circ} \mathrm{C}$.

\section{Confocal, immuno-electron microscopy (immuno-EM), and total internal reflection fluorescence (TIRF) imaging}

For confocal analysis, cells $\left(1 \times 10^{5}\right)$ were fixed and then incubated with primary antibodies against Rab37 and TIMP1. After incubation with secondary fluorescent antibodies, the images were obtained using Olympus FV1000 confocal microscope. For immuno-EM analysis, primary antibodies and the conjugated 10 or $15 \mathrm{~nm}$ gold particles were listed in Supplementary Table 2. For TIRF analysis, cells expressing WT-Rab37 were transiently transfected with GFP-TIMP1 for $24 \mathrm{~h}$ before image analysis. To capture the Rab37 trafficking events, we tracked each vesicle trafficking distance with trackIT software (Olympus, Tokyo, Japan). 


\section{Immunoblotting}

Cells were lysed in $1 \times$ RIPA lysis buffer with a cocktail of antiproteases (Sigma-Aldrich, MO, USA), equal amounts of total cellular extracts were electrophoresed by SDS-PAGE and transferred on to a polyvinylidene difluoride membrane. Primary antibodies used for immunoblotting are list in Supplementary Table 2.

\section{Migration and invasion assays}

For transwell migration and invasion assays, $1 \times 10^{5}$ cells were seeded onto the upper chamber with or without Matrigel (Sigma-Aldrich, MO, USA) and cultured for $16 \mathrm{~h}$. Cells attached on the reverse side of the membrane were then fixed and stained. Ten random views were photographed and quantified.

\section{Experimental metastasis assays in vivo}

BALB/c nude mice were purchased from Laboratory Animal Center of National Cheng Kung University. All experimental procedures involving the use and care of mice were approved by the Institutional Animal Care and Use Committee of National Cheng Kung University (\#104165). All protocols were performed according to the guidelines of "Law Concerning the Protection and Control of Animals" and "Animal Protection Act". Mice were divided into three groups including control EV cells, WT-Rab37 cells, and T172D-Rab37 cells. Cells were resuspended in serum-free medium $\left(2 \times 10^{6}\right.$ cells per 200 $\mathrm{ml}$ ) and injected into BALB/c nude mice via the tail vein. Mice were sacrificed after 6 weeks and lung tissues were resected, fixed and embedded in paraffin for histological H\&E and immunohistochemistry staining using indicated antibodies (Supplementary Table 2).

\section{Clinical samples and information}

We recruited 158 lung cancer patients from National Cheng Kung University Hospital (NCKUH), Taiwan after obtaining appropriate institutional review and informed consent from patients. This study was approved by the Institutional Review Board of NCKUH (B-ER-103-369). All clinical samples used were conducted in accordance with the guidelines of the Declaration of Helsinki. Paraffin sections were collected for immunohistochemistry analysis. Overall survival was calculated from the day of surgery to the date of death or the last follow-up. The end of the follow-up period was defined as June 2016. The mean follow-up period was 64.8 months (range 9-103 months).

\section{Statistical analysis}

Statistical analysis was performed by Student's $t$ test. Data shown were representatives of at least three independent experiments unless indicated otherwise. Pearson's $\chi^{2}$ test was used to compare the correlation of between PKC $\alpha$, TIMP1 and Rab37 expression levels and their association with clinicopatholgical parameters in lung cancer patients. Overall survival curves were calculated according to the Kaplan-Meier analysis. Cox regression comparison was performed to analyze the relative risk for poor outcome in patients. Data represented mean \pm SD. $P<0.05$ was considered to be statistically significant.

\section{Abbreviations}

TIMP1, tissue inhibitor of metalloproteinases 1; PKC $\alpha$, protein kinase $\mathrm{C} \alpha$; TSP1, thrombospondin-1; CIP, calf intestinal phosphatase; PMA, phorbol 12-myristate 13-acetate; CM, conditioned medium; EM, electron microscopy; TIRF, total internal reflection fluorescence; GAP, GTPase-activating proteins; GEF, guanine nucleotide exchange factors.

\section{Author contributions}

Y.C.W. and C.W.C conceived the project idea, designed the studies, and supervised all aspects of the project. H.T.T., T.H.L. and Y.A.T. designed and carried experiments and analyzed data. C.H.T. performed and analyzed cell experiments. P.J.L and W.W.L. provided clinical samples. Y.C.W. and H.T.T. wrote the manuscript with edits from all authors. Y.C.W. and C.W.C. obtained funding.

\section{ACKNOWLEDGMENTS}

We thank the transmission electron microscope core at National Cheng Kung University for technical support. We acknowledge Dr. Hsiao-Sheng Liu (Department of Microbiology and Immunology at National Cheng Kung University, Taiwan) and Dr. Hung-Chi Cheng (Department of Biochemistry and Molecular Biology at National Cheng Kung University, Taiwan) for help and advice throughout this work.

\section{CONFLICTS OF INTEREST}

No potential conflicts of interest were disclosed.

\section{FUNDING}

This work was supported in part by grants MOST105-2320-B-006-007, NHRI-EX104-10202BI, and MOHW105-TDU-B-211-124-003 (Y-C Wang) and by grants from the Ministry of Education, Taiwan, R. O. C. and the aim for the Top University Project to the National Cheng Kung University (C-W Chiang). 


\section{REFERENCES}

1. Stenmark H. Rab GTPases as coordinators of vesicle traffic. Nat Rev Mol Cell Biol. 2009; 10:513-525.

2. Zerial M, McBride $H$. Rab proteins as membrane organizers. Nat Rev Mol Cell Biol. 2001; 2:107-117.

3. Goldenring JR. A central role for vesicle trafficking in epithelial neoplasia: intracellular highways to carcinogenesis. Nat Rev Cancer. 2013; 13:813-820.

4. Bhuin T, Roy JK. Rab proteins: the key regulators of intracellular vesicle transport. Exp Cell Res. 2014; 328:1-19.

5. Segawa T, Hazeki K, Nigorikawa K, Morioka S, Guo Y, Takasuga S, Asanuma K, Hazeki O. Inpp5e increases the Rab5 association and phosphatidylinositol 3-phosphate accumulation at the phagosome through an interaction with Rab20. Biochem J. 2014; 464:365-375.

6. Hayes GL, Brown FC, Haas AK, Nottingham RM, Barr FA, Pfeffer SR. Multiple Rab GTPase binding sites in GCC185 suggest a model for vesicle tethering at the trans-Golgi. Mol Biol Cell. 2009; 20:209-217.

7. Pfeffer SR. Structural clues to Rab GTPase functional diversity. J Biol Chem. 2005; 280:15485-15488.

8. Luo ML, Gong C, Chen $\mathrm{CH}$, Hu H, Huang P, Zheng M, Yao Y, Wei S, Wulf G, Lieberman J, Zhou XZ, Song E, Lu KP. The Rab2A GTPase promotes breast cancer stem cells and tumorigenesis via Erk signaling activation. Cell Rep. 2015; 11:111-124.

9. Yang J, Liu W, Lu X, Fu Y, Li L, Luo Y. High expression of small GTPase Rab3D promotes cancer progression and metastasis. Oncotarget. 2015; 6:11125-38. https://doi. org/10.18632/oncotarget.3575.

10. Bravo-Cordero JJ, Marrero-Diaz R, Megías D, Genís L, García-Grande A, García MA, Arroyo AG, Montoya MC. MT1-MMP proinvasive activity is regulated by a novel Rab8dependent exocytic pathway. EMBO J. 2007; 26:1499-1510.

11. Yoon SO, Shin S, Mercurio AM. Hypoxia stimulates carcinoma invasion by stabilizing microtubules and promoting the Rab11 trafficking of the alpha6beta4 integrin. Cancer Res. 2005; 65:2761-2769.

12. Pellinen T, Arjonen A, Vuoriluoto K, Kallio K, Fransen JA, Ivaska J. Small GTPase Rab21 regulates cell adhesion and controls endosomal traffic of beta1-integrins. J Cell Biol. 2006; 173:767-780.

13. Hou Q, Wu YH, Grabsch H, Zhu Y, Leong SH, Ganesan K, Cross D, Tan LK, Tao J, Gopalakrishnan V, Tang BL, Kon OL, Tan P. Integrative genomics identifies RAB23 as an invasion mediator gene in diffuse-type gastric cancer. Cancer Res. 2008; 68:4623-4630.

14. Cheng KW, Lahad JP, Kuo WL, Lapuk A, Yamada K, Auersperg N, Liu J, Smith-McCune K, Lu KH, Fishman D, Gray JW, Mills GB. The RAB25 small GTPase determines aggressiveness of ovarian and breast cancers. Nat Med. 2004; 10:1251-1256.
15. Hendrix A, Maynard D, Pauwels P, Braems G, Denys H, Van den Broecke R, Lambert J, Van Belle S, Cocquyt V, Gespach C, Bracke M, Seabra MC, Gahl WA, et al. Effect of the secretory small GTPase Rab27B on breast cancer growth, invasion, and metastasis. J Natl Cancer Inst. 2010; 102: 866-880.

16. Wheeler DB, Zoncu R, Root DE, Sabatini DM, Sawyers CL. Identification of an oncogenic RAB protein. Science. 2015; 350:211-217.

17. Wu CY, Tseng RC, Hsu HS, Wang YC, Hsu MY. Frequent down-regulation of hRAB37 in metastatic tumor by genetic and epigenetic mechanisms in lung cancer. Lung Cancer. 2009; 63:360-367.

18. Tsai CH, Cheng HC, Wang YS, Lin P, Jen J, Kuo IY, Chang YH, Liao PC, Chen RH, Yuan WC, Hsu YS, Yang MH, Hsu MT, et al. Small GTPase Rab37 targets tissue inhibitor of metalloproteinase 1 for exocytosis and thus suppresses tumour metastasis. Nat Commun. 2014; 5:4804.

19. Tzeng HT, Tsai CH, Yen YT, Cheng HC, Chen YC, Pu SW, Wang YS, Shan YS, Tseng YL, Su WC, Lai WW, Wu LW, Wang YC. Dysregulation of Rab37-mediated cross-talk between cancer cells and endothelial cells via thrombospondin-1 promotes tumor neovasculature and metastasis. Clin Cancer Res. 2017; 23:2335-2345.

20. Ong ST, Freeley M, Skubis-Zegadło J, Fazil MH, Kelleher D, Fresser F, Baier G, Verma NK, Long A. Phosphorylation of Rab5a protein by protein kinase $\mathrm{C} \varepsilon$ is crucial for T-cell migration. J Biol Chem. 2014; 289:19420-19434.

21. Pavarotti M, Capmany A, Vitale N, Colombo MI, Damiani MT. Rab11 is phosphorylated by classical and novel protein kinase $\mathrm{C}$ isoenzymes upon sustained phorbol ester activation. Biol Cell. 2012; 104:102-115.

22. Fitzgerald ML, Reed GL. Rab6 is phosphorylated in thrombin-activated platelets by a protein kinase C-dependent mechanism: effects on GTP/GDP binding and cellular distribution. Biochem J. 1999; 342:353-360.

23. Cherfils J, Zeghouf M. Regulation of small GTPases by GEFs, GAPs, and GDIs. Physiol Rev. 2013; 93:269-309.

24. Scheffzek K, Ahmadian MR, Wittinghofer A. GTPaseactivating proteins: helping hands to complement an active site. Trends Biochem Sci. 1998; 23:257-262.

25. Ayad N, Hull M. Mellman I. Mitotic phosphorylation of rab4 prevents binding to a specific receptor on endosome membranes. EMBO J. 1997; 16:4497-4507.

26. van der Sluijs P, Hull M, Huber LA, Mâle P, Goud B, Mellman I. Reversible phosphorylation--dephosphorylation determines the localization of rab4 during the cell cycle. EMBO J. 1992; 11:4379-4389.

27. Lai YC, Kondapalli C, Lehneck R, Procter JB, Dill BD, Woodroof HI, Gourlay R, Peggie M, Macartney TJ, Corti O, Campbell DG, Itzen A, Trost M, et al. Phosphoproteomic screening identifies Rab GTPases as novel downstream targets of PINK1. EMBO J. 2015; 34:2840-2861. 
28. Kettenbach AN, Schweppe DR, Faherty BK, Pechenick D, Pletnev AA, Gerber SA. Quantitative phosphoproteomics identifies substrates and functional modules of Aurora and Polo-like kinase activities in mitotic cells. Sci Signal. 2011; 4:rs5.

29. Blethrow JD, Glavy JS, Morgan DO, Shokat KM. Covalent capture of kinase-specific phosphopeptides reveals Cdk1cyclin B substrates. Proc Natl Acad Sci USA. 2008; 105:1442-1447.

30. Johnson LN. Substrates of mitotic kinases. Sci Signal. 2011; 4:pe31.

31. Duarte ML, Pena DA, Nunes Ferraz FA, Berti DA, Paschoal Sobreira TJ, Costa-Junior HM, Abdel Baqui MM, Disatnik MH, Xavier-Neto J, Lopes de Oliveira PS, Schechtman D. Protein folding creates structure-based, noncontiguous consensus phosphorylation motifs recognized by kinases. Sci Signal. 2014; 7:ra105.

32. Nakashima S. Protein kinase C alpha (PKC alpha): Regulation and biological function. J Biochem. 2002; 132:669-675.

33. Qin L, Yin YT, Zheng FJ, Peng LX, Yang CF, Bao YN, Liang YY, Li XJ, Xiang YQ, Sun R, Li AH, Zou RH,
Pei XQ, et al. WNT5A promotes stemness characteristics in nasopharyngeal carcinoma cells leading to metastasis and tumorigenesis. Oncotarget. 2015; 6:10239-52. https://doi. org/10.18632/oncotarget.3518.

34. Kyuno D, Kojima T, Yamaguchi H, Ito T, Kimura Y, Imamura M, Takasawa A, Murata M, Tanaka S, Hirata K, Sawada N. Protein kinase $\mathrm{C} \alpha$ inhibitor protects against downregulation of claudin-1 during epithelial-mesenchymal transition of pancreatic cancer. Carcinogenesis. 2013; 34:1232-1243.

35. Wu B, Zhou $\mathrm{H}, \mathrm{Hu} \mathrm{L}, \mathrm{Mu} \mathrm{Y}, \mathrm{Wu}$ Y. Involvement of $\mathrm{PKC} \alpha$ activation in TF/VIIa/PAR2-induced proliferation, migration, and survival of colon cancer cell SW620. Tumour Biol. 2013; 34:837-846.

36. Walsh MF, Woo RK, Gomez R, Basson MD. Extracellular pressure stimulates colon cancer cell proliferation via a mechanism requiring PKC and tyrosine kinase signals. Cell Prolif. 2004; 37:427-441. 